\title{
En anticipación de una vacuna antirrotavirus: revisión de estudios epidemiológicos sobre la diarrea por rotavirus en la Argentina ${ }^{1}$
}

\author{
Jorge A. Gómez, ${ }^{2}$ Silvia Nates, ${ }^{3}$ Nelda R. de Castagnaro, 4 \\ Carlos Espul, ${ }^{5}$ Ana Borsa ${ }^{6}$ y Roger I. Glass ${ }^{7}$
}

RESUMEN En todo el mundo, los rotavirus son la causa más común de diarrea grave en los niños pequeños y actualmente se están ensayando sobre el terreno vacunas que posiblemente permitan inmunizar a la población infantil dentro de varios años. Con el fin de estimar la carga de enfermedad por rotavirus en la Argentina y la utilidad de establecer en el país un sistema de vigilancia de la enfermedad, se revisaron datos sobre la detección de estos virus, según estudios publicados y otros inéditos de nueve ciudades argentinas y uno multicéntrico. Los informes revisados indican que se detectaron rotavirus en 20\% de 5226 especímenes (con un recorrido de 6 a 54\% entre estudios) tomados de niños hospitalizados por diarrea y en 9\% de 6587 especímenes (recorrido de 5 a 22\% entre estudios) tomados de pacientes ambulatorios, miembros de poblaciones mixtas (niños hospitalizados y ambulatorios) y sujetos de encuestas comunitarias. Los datos hospitalarios muestran que, si bien los virus se detectaron durante todo el año, en los meses de invierno (mayo a julio) hubo un pico de intensidad cuando hasta la mitad de los niños con diarrea dieron resultados positivos a los rotavirus. En tres laboratorios se logró serotipificar para la proteína G 230 de 294 especímenes positivos (78\%); los resultados indican que el serotipo G1 fue el más común (presente en $60 \%$ de los especímenes serotipificados) seguido del G2 (en 20\%), G4 (en 14\%) y G3 (en 5\%). Sobre la base de los datos obtenidos en el país, se estimó que en 1991 en la Argentina hubo aproximadamente 84500 visitas de pacientes ambulatorios ( 1 de cada 8 nacimientos) y 21000 hospitalizaciones de 4 dias en promedio (1 de cada 31 nacimientos) asociadas con rotavirus, que en conjunto costaron unos US\$27,7 millones. Estos datos preliminares muestran que la carga de enfermedad por rotavirus en los niños argentinos es muy pesada y podría disminuirse con una vacuna segura y efectiva. Además se necesita ampliar la vigilancia para mejorar el conocimiento de la epidemiología y de la distribución de las cepas de rotavirus en el país, calcular más exactamente la eficacia en función del costo de un programa de vacunación antirrotavirus y determinar los mejores métodos de monitorear sus efectos.

\footnotetext{
Se publicó en inglés en esta revista (Vol. 3, No. 2, 1998, pp. 69-77) con el título "Anticipating rotavirus vaccines: review of epidemiologic studies of rotavirus diarrhea in Argentina".

2 Ministerio de Salud y Acción Social de la Nación, Instituto Nacional de Enfermedades Infecciosas, Buenos Aires, Argentina. Dirección postal: Departamento de Virología, Instituto Nacional de Enfermedades Infecciosas, Av. Vélez Sarsfield 563 (1281), Buenos Aires, Argentina. Teléfono 54-1301-7428; fax 54-1-303-2382.
}

3 Universidad Nacional de Córdoba, Instituto de Virología “Dr. J.M. Vanella", Córdoba, Argentina.

4 Universidad Nacional de Tucumán, Instituto de Microbiología, Tucumán, Argentina.

5 Hospital Central de Mendoza, Laboratorio de Virología, Mendoza, Argentina.

6 Hospital de Niños de La Plata, Laboratorio de Virología, Buenos Aires, Argentina.

7 Centros para el Control y la Prevención de Enfermedades, Sección de Gastroenteritis Virales, Atlanta, GA, EUA.
La diarrea constituye una de las afecciones infantiles más corrientes en el mundo entero. En los países en desarrollo se ha estimado que la diarrea aguda causa de 3,5 a 4,0 millones de muertes al año (1). En 1993, las autoridades sanitarias de la Argentina notificaron más de 400 muertes asocia- 
das con diarreas sin causa específica (infecciones intestinales mal definidas, código 009 de la C.I.E.) (I. Miceli, comunicación personal, 1995). Asimismo, la diarrea en los niños es causa importante tanto de hospitalización como de visitas al médico, pero su número nunca ha sido cuantificado ni se han documentado bien los agentes etiológicos.

Tras el descubrimiento de los rotavirus por Bishop et al. en 1973 (2), se ha documentado en muchos estudios epidemiológicos que los rotavirus son los agentes causales más importantes de diarrea grave en niños en el mundo entero. La mayoría de las infecciones primarias por rotavirus están asociadas con diarreas agudas que pueden conducir a la deshidratación y, ocasionalmente, a la muerte. Se ha calculado que a los rotavirus se atribuyen de 20 a $40 \%$ de las hospitalizaciones asociadas con diarrea en los niños y unas 870000 defunciones al año, en particular en los países en desarrollo (3). Aun en un país industrializado como los Estados Unidos, donde la mortalidad debida a diarreas por rotavirus es relativamente poca (20 a 40 defunciones al año), la morbilidad resulta elevada: cada año se hospitaliza por rotavirus a unos 55000 niños, a un costo estimado de US\$ 1400 millones entre gastos médicos y costos indirectos (4).

Ciertos estudios epidemiológicos iniciales sugirieron que los rotavirus se podrían combatir con vacunas. Varias encuestas longitudinales demostraron una inmunidad natural; los niños infectados más de una vez presentaban síntomas menos graves cuando se reinfectaban (5-7) y los neonatos adquirían una protección que duraba varios años $(8,9)$. No obstante, la inmunidad conferida por la infección natural no ofrece protección completa y los adultos seropositivos pueden sufrir diarreas por rotavirus durante la atención de niños enfermos o cuando viajan a países en desarrollo, donde las infecciones entéricas son corrientes (10).

La elaboración de una vacuna antirrotavírica fue facilitada por el descubrimiento de métodos para el cultivo de rotavirus humanos en cultivos de tejidos (11). Se han creado diversas vacunas vivas atenuadas y se están ensayando dos vacunas orales vivas sobre el terreno $(12,13)$. Una vez aprobadas, se considerará la posibilidad de utilizarlas como parte de la inmunización rutinaria de los niños en los Estados Unidos. En ese contexto, una nueva vacuna podría causar una disminución muy marcada del número de visitas médicas y hospitalizaciones por diarreas infantiles y podría reducir el número de defunciones asociadas con diarreas en los países en desarrollo.

En la Argentina se detectaron rotavirus como causa de gastroenteritis infantil por primera vez en 1975, cuando Lombardi et al. descubrieron rotavirus en 20 muestras fecales diarreicas examinadas por microscopía electrónica (14). En 1978, Muchinik et al. realizaron el primer estudio para determinar la frecuencia de rotavirus en pacientes con diarrea aguda que ingresaron al Hospital de Niños "Ricardo Gutiérrez" de Buenos Aires $(15,16)$. Desde entonces, una serie de estudios adicionales realizados por varios grupos han evaluado distintos aspectos de la epidemiología de los rotavirus en diferentes medios (17-35).

En 1995, la Sociedad Argentina de Virología organizó un taller sobre gastroenteritis víricas en el Instituto $\mathrm{Na}$ cional de Microbiología "Dr. Carlos G. Malbrán", con el fin de examinar la carga de las enfermedades por rotavirus, debatir las medidas que se debían tomar y proponer nuevas estrategias para su control en el futuro. Este taller recomendó la organización de lo que posteriormente se llamó el Grupo para el Estudio de las Diarreas Virales, encargado de examinar toda la información publicada e inédita acerca de la epidemiología de los rotavirus en la Argentina y de realizar una encuesta nacional sobre las diarreas por rotavirus, utilizando métodos de diagnóstico estándar y un diseño común para todo el estudio. El presente artículo presenta nuestra primera revisión de los estudios ya realizados en la Argentina. En él se examina la epidemiología de las diarreas por rotavirus, evaluar la carga atribuible a la enfermedad e identificar mejores métodos para vigi- lar su epidemiología en caso de que se iniciara un programa nacional de vacunación.

\section{MATERIALES Y MÉTODOS}

Examinamos datos de 17 estudios realizados en seis ciudades de la Argentina (Avellaneda, Buenos Aires, Córdoba, La Plata, Mendoza y Tucumán), observaciones en tres ciudades (descripciones de rotavirus no pertenecientes al grupo A en Resistencia y Posadas y un estudio epidemiológico de 5 meses de duración en Jujuy (36)); y datos arrojados por un proyecto multicéntrico (37) realizado en las ciudades de Buenos Aires, Córdoba, La Plata, Mar del Plata, Posadas, Rosario y Tucumán (figura 1 y cuadro 1 ). Este estudio multicéntrico, diseñado especialmente para obtener información sobre las diarreas bacterianas, aportó ciertos resultados sobre la epidemiología de los rotavirus humanos que no coincidían con los otros resultados ya analizados. En la mayoría de los estudios epidemiológicos que se revisaron, se detectó un rotavirus utilizando ensayos inmunoenzimáticos (EIE) $(21-26,29,30,35,38)$ o análisis del ARN vírico por electroforesis en gel de poliacrilamida (PAGE) (18-20, 25-34, 36, 39). Los procedimientos PAGE utilizados fueron similares en todos los estudios y tenían una sensibilidad comparable a la de los EIE, con positividad en $92 \%$ (26) y $100 \%$ (32) de las muestras que dieron resultados positivos en el EIE. Los laboratorios que participaron en el estudio multicéntrico (37) utilizaron distintos métodos de detección de rotavirus (PAGE, ensayo de inmunoabsorción enzimática [ELISA], y aglutinación en látex). Los estudios iniciales de Muchinik et al. $(15,16)$ utilizaron la microscopía electrónica y la inmunoelectroforesis de contraste, que son un poco menos sensibles que los métodos de EIE y PAGE.

Revisamos los distintos estudios para identificar las tendencias anuales y estacionales en la detección de los rotavirus, las diferencias en las tasas de detección entre poblaciones hospi- 
FIGURA 1. Tasas de detección de rotavirus en la Argentina notificadas en algunos de los estudios que se enumeran en el cuadro 1 sobre niños con diarreas (se incluyen estudios sobre casos hospitalizados y ambulatorios y una combinación de ambos)

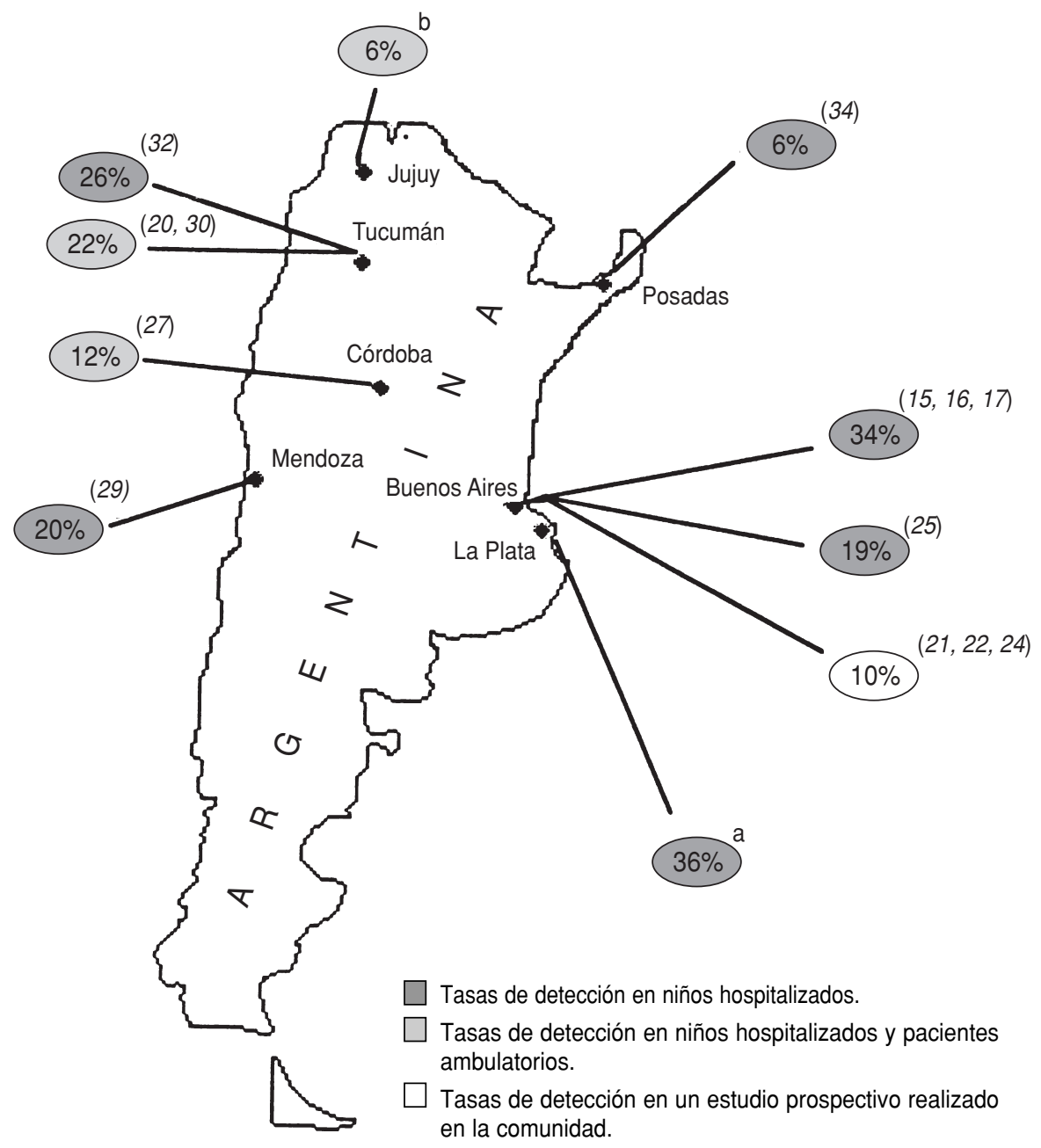

a Borsa A. Comunicación personal, julio de 1995.

${ }^{b}$ Caillou MSL, Komaid JA, Zamora AM, Figueroa T, Castagnaro NCR. Comparación de los patrones electroforéticos de rotavirus detectados en Tucumán y Jujuy. Informe inédito, agosto de 1991.

talizadas y no hospitalizadas, y la distribución de los pacientes por edades. También se usaron datos compuestos sobre hospitalizaciones y visitas a servicios ambulatorios para estimar la carga atribuible a las diarreas por rotavirus en la Argentina y el costo que representa la enfermedad para el sistema de atención de la salud. En distintos estudios de laboratorio se identificaron los serotipos $\mathrm{G}$ predominantes del grupo de los rotavirus mediante la utilización de un EIE con base monoclonal anteriormente descrito (40). Ciertos rotavirus del grupo $C$, cepa antigénicamente distinta y con rasgos epidemiológicos diferentes, fueron hallados en cuatro centros en que se utilizó el PAGE como método diagnóstico de rutina.

\section{RESULTADOS}

De los 17 estudios incluidos en el análisis, 10 se hicieron en pacientes hospitalizados por diarrea y seis en pacientes ambulatorios o poblaciones mixtas (pacientes ingresados y ambulatorios), y en uno se examinó la prevalencia de la infección por rotavirus en neonatos asintomáticos (cuadro 1). Solo dos de estos estudios efectuaron una vigilancia de más de 3 años y recibieron muestras tanto de niños hospitalizados como de niños tratados por consulta externa $(20,27)$. Un estudio comunicó la frecuencia mensual de la identificación de rotavirus (20). El único estudio en neonatos asintomáticos identificó la presencia de rotavirus en 3\% de 304 niños que nacieron en una clínica obstétrica de Tucumán entre mayo y octubre de 1990 (32).

\section{Los rotavirus en niños hospitalizados}

Tal como se indica en el cuadro 1, 10 estudios comunicaron la frecuencia de la detección de rotavirus en niños hospitalizados por diarrea $(14-17,25$, $29,31,32,34,35,37,38)$. La tasa mediana de detección de rotavirus fue de $20 \%$ (intervalo de confianza de $95 \%$ [IC95\%]: $14 \%$ a $34 \%$ ) y las cifras obtenidas en los distintos estudios oscilaron entre 6 y 54\%. Los dos informes inéditos indicaban frecuencias anuales de $11 \%$ (37) y $36 \%$ (38). Las diferentes tasas de detección no estaban claramente asociadas con los distintos métodos de detección utilizados en los estudios.

\section{Los rotavirus en otros niños}

En un total de seis estudios se examinó la frecuencia de la detección de rotavirus en pacientes ambulatorios con diarrea o en miembros de la comunidad. De estos estudios, dos examinaron la frecuencia de diarrea por rotavirus en pacientes pediátricos ambulatorios $(35,37)$; otros tres se realizaron en poblaciones mixtas de niños hospitalizados y ambulatorios con diarrea $(20,27,30,33,36)$ y uno fue un estudio prospectivo de tipo comunitario que se realizó en la ciudad de Avellaneda, $15 \mathrm{~km}$ al sur de Buenos Aires 
CUADRO 1. Estudios epidemiológicos sobre los rotavirus en niños de la Argentina. Se indican el tipo de sujeto examinado (niños hospitalizados y tratados ambulatoriamente, con o sin diarrea); ciudad y año en que se hizo el estudio; recorrido de edades; tipo de ensayo detector de rotavirus; número de muestras examinadas, y número y porcentaje de muestras positivas a rotavirus

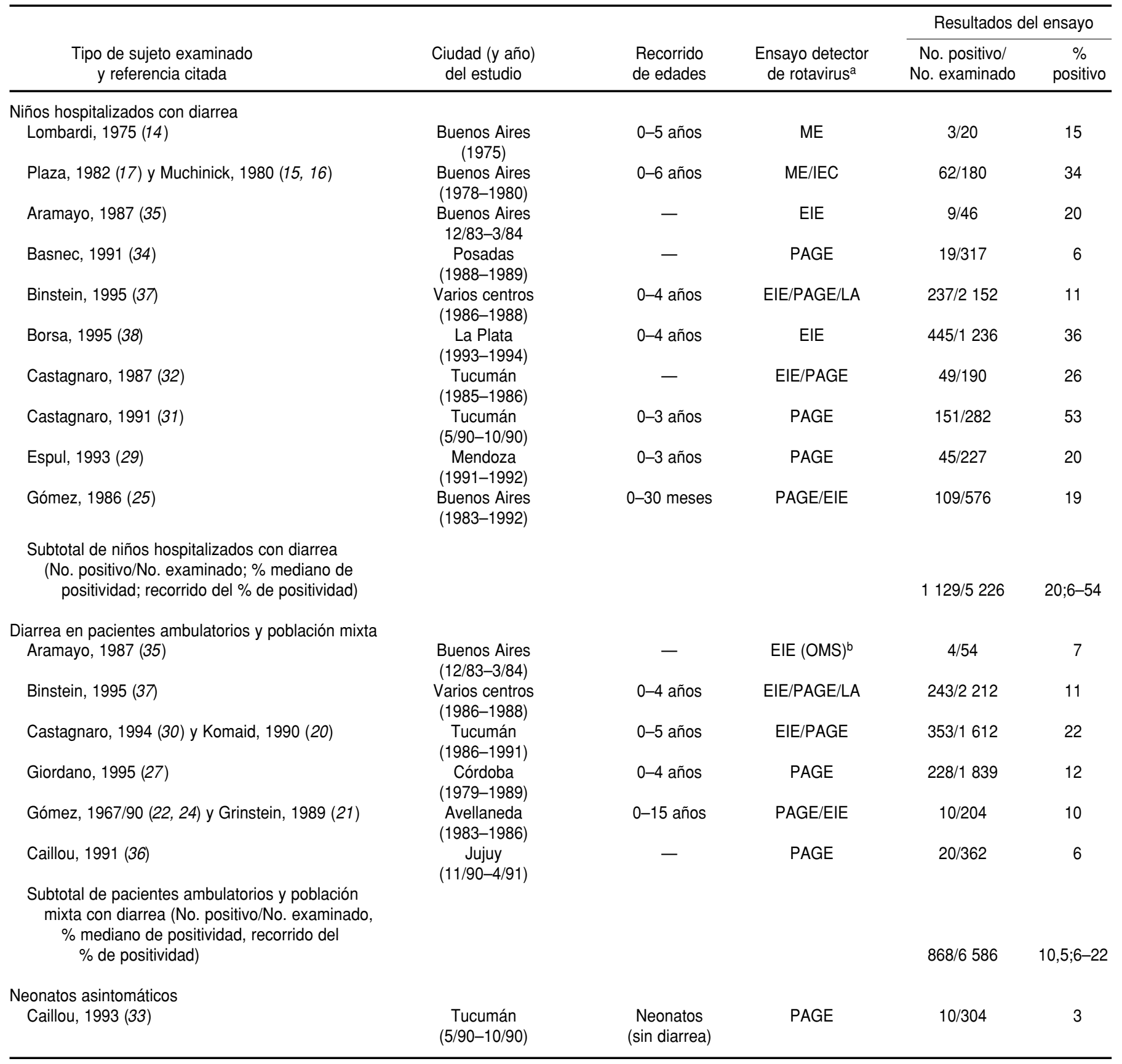

a ME: microscopía electrónica; IEC: inmunoelectroforesis de contraste; EIE: ensayo inmunoenzimático; PAGE: electroforesis en gel de poliacrilamida; AL: aglutinación en látex.

${ }^{b}$ ELISA formulado y distribuido por el Collaborating Center for Reference and Research on Rotavirus, de la OMS, Birmingham, Reino Unido.

$(21,22,24)$. El estudio multicéntrico de Binstein y sus colegas (inédito) fue organizado concretamente para identificar agentes bacterianos causantes de diarrea, pero se detectaron rotavirus en $11 \%$ de las muestras fecales de más de 4000 casos examinados entre 1986 y 1989. El otro estudio que aportó datos sobre pacientes pediátricos ambulatorios (y que mantuvo separados estos datos de los correspondientes a niños hospitalizados) procuró identificar todos los agentes patógenos que fueron causa reconocida de diarrea durante la estación estival en el Hospi- 
tal de Niños de Buenos Aires. Este estudio determinó rotavirus con una frecuencia de $7 \%$ en los pacientes ambulatorios y de $26 \%$ en los niños hospitalizados (35). En los tres estudios en poblaciones mixtas (que comprendían pacientes externos y niños hospitalizados) se detectaron rotavirus en $22 \%$ (20), $12 \%$ (27) y $6 \%$ (36) de los niños incluidos en los estudios.

Por último, en el estudio comunitario de la ciudad de Avellaneda se identificaron rotavirus en $10 \%$ de los pacientes con diarrea aguda $(21,22,24)$.

La tasa mediana de detección de rotavirus en los grupos heterogéneos examinados en los seis estudios fue de $10,5 \%$ (IC95\%: $5 \%$ a $17 \%$ ) y las tasas obtenidas en los distintos estudios oscilaron entre 6 y 22\% (cuadro 1). En general, estos estudios parecen sugerir que es más bajo el porcentaje de diarreas asociadas con rotavirus en poblaciones de pacientes ambulatorios que en pacientes hospitalizados.

\section{Tendencias estacionales}

La incidencia estacional de las diarreas por rotavirus se examinó en tres de los estudios que duraron 2 años o más (figura 2a). Se detectaron los mayores porcentajes de pacientes con rotavirus durante los meses fríos de mayo a julio $(20,21,24,25)$, cuando hubo ocasiones en que se identificaron en más de $50 \%$ de todos los niños hospitalizados por diarrea $(15-17,31,32)$. Tanto en Buenos Aires como en Tucumán la detección de rotavirus tuvo un pico invernal cuando el número total de casos de diarrea era relativamente bajo; los casos de diarrea culminaban en el verano y en su gran mayoría eran de origen bacteriano (figura 2b) (21).

Un estudio prospectivo sobre la diarrea infantil realizado en la comunidad de Avellaneda, cerca de Buenos Aires, demostró que la incidencia de diarreas asociadas con rotavirus era significativamente mayor durante los meses invernales (de mayo a julio) (8,8 casos por 1000 meses-persona) que en el resto del año (tres casos por 1000 meses-persona) (21,24). Si bien el porcentaje máximo de diarreas causa-
FIGURA 2. Frecuencia de diarreas y de diarreas por rotavirus en la Argentina, según la estación del año. Se destaca que los episodios diarreicos mostraron la tendencia a tener su número máximo en los meses de verano, mientras que la proporción de casos causados por rotavirus alcanzó su máxima frecuencia en los meses de invierno
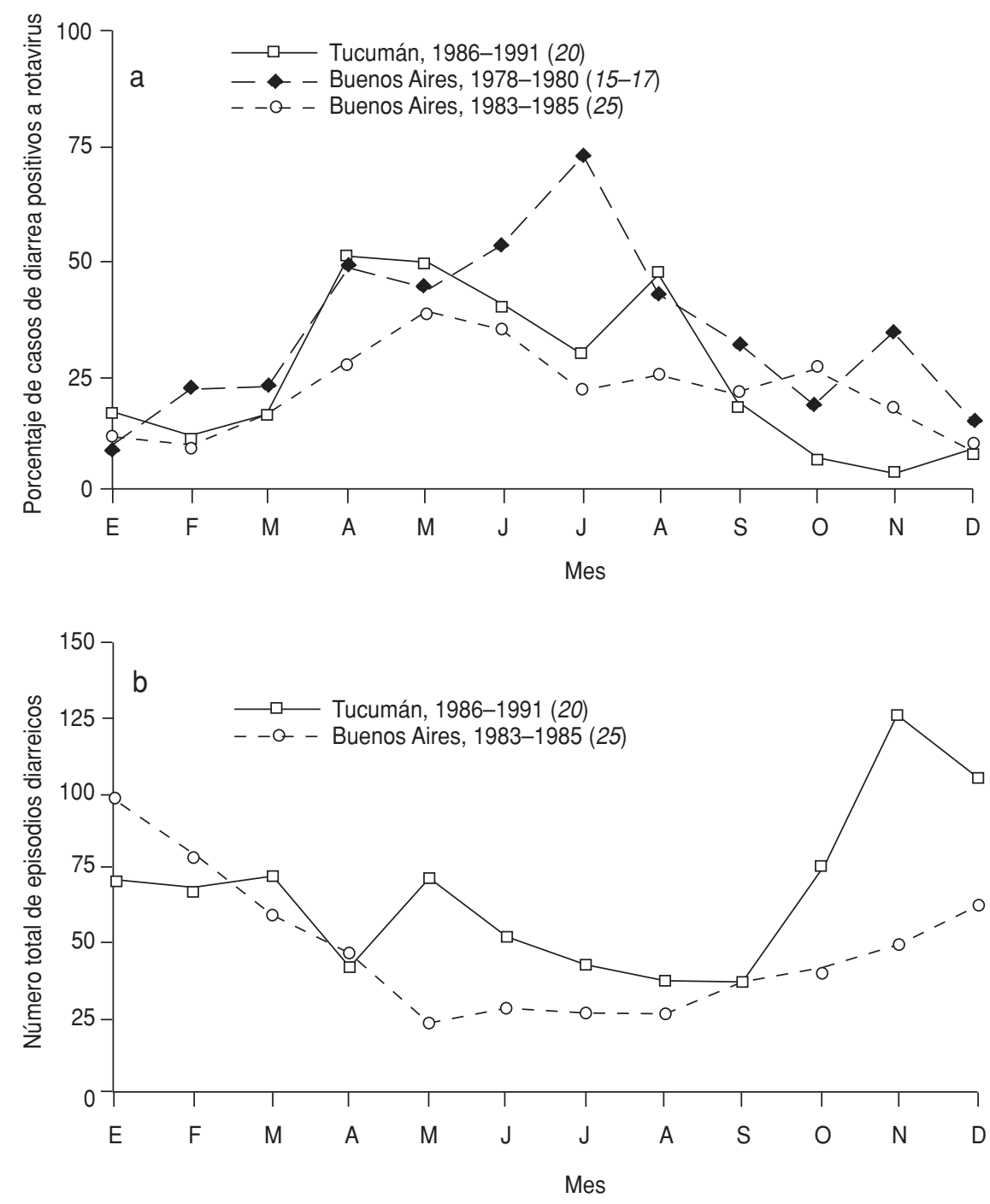

das por rotavirus ciertamente se ve influido por la disminución del número de casos de diarrea observados en los meses de invierno, los dos estudios que arrojaron información pormenorizada sobre varios años también pusieron de manifiesto un mayor número de casos de rotavirus en los meses invernales de mayo a julio $(20,21,24)$.

\section{Edad de los pacientes}

Aunque en todas partes del mundo los rotavirus suelen causar diarreas en niños de 6 a 24 meses de edad, en la Argentina los niños tienden a sufrir diarreas por rotavirus a una edad relativamente temprana (figura 3). Algunos investigadores notificaron que $80 \%$ de los rotavirus se detectaron durante el primer año de vida y que $35 \%$ de ellos se producían antes de los 6 meses de edad $(17,21,24)$. Por otra parte, el estudio multicéntrico inédito de Binstein y sus colegas no mostró declinación alguna de las tasas de detección de diarrea por rotavirus entre el nacimiento y los 4 años de edad. 
FIGURA 3. Distribución según edad de los casos de diarrea por rotavirus notificados en estudios realizados en Tucumán (31), Avellaneda $(21,22,24)$ y Buenos Aires (15-17)

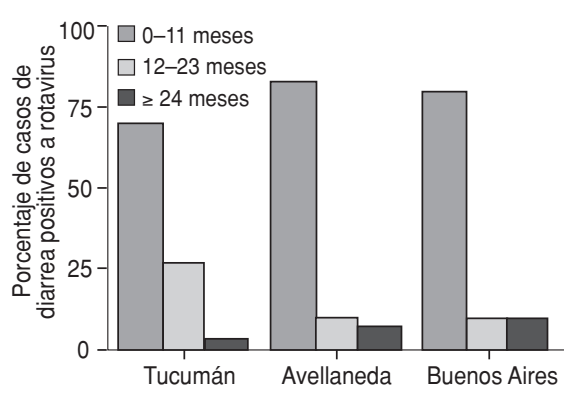

\section{Serotipos rotavíricos}

Un total de 294 cepas de rotavirus de tres series separadas de tomas de muestras fueron sometidas a ELISA para serotipificar su proteína-G (es decir, la proteína VP7 de la cápsida vírica externa [cuadro 2]). De estas 294 cepas, 123 se obtuvieron en Buenos Aires (1983-1985) (23), 126 fueron aisladas en Tucumán (1986-1991) (30) y 45 se obtuvieron en Mendoza (19911992) (29). No fue posible establecer el serotipo de las cepas encontradas en todas las muestras, pero de las 230 $(78 \%)$ en que sí fue posible, $137(60 \%)$ correspondían al serotipo G1, $46(20 \%)$ al serotipo G2, 32 (14\%) al G4, 11 (5\%) al G3, y $4(2 \%)$ a serotipos distintos de los G1-G4. Tal como se indica en el cuadro, todas las cepas de Mendoza menos una eran G1 y no se detectaron cepas G3 en las muestras de Tucumán.

El estudio de Buenos Aires fue el único en que se utilizó un anticuerpo monoclonal dirigido a un antígeno común en la proteína VP7, además de cuatro anticuerpos monoclonales para la serotipificación. En 46 de las 50 muestras de Buenos Aires en que no se pudo determinar el serotipo, el espécimen no mostró reactividad frente al anticuerpo usado con anterioridad, lo cual sugiere que no fue posible determinar el serotipo debido a la degradación del espécimen. Tres de los cuatro especímenes restantes que no se pudieron tipificar mostraron reactividad a más de uno de los anticuerpos monoclónicos usados para la serotipificación, mientras que el último espécimen respondió de forma negativa a todos los cuatro anticuerpos. No se realizaron análisis de los serotipos $\mathrm{P}$ de cepas argentinas.

\section{Rotavirus del grupo C}

Se identificaron mediante electroforesis en gel de poliacrilamida (PAGE) siete cepas de rotavirus del grupo en muestras obtenidas en Resistencia (19), Posadas (34), Tucumán (18) y Buenos Aires (39). Sin embargo, ninguna de estas cepas ha sido confirmada con reactivos específicos para los rotavirus del grupo C. Este hallazgo fortuito en cuatro localidades separadas sugiere que los rotavirus del grupo $C$ tal vez tengan una amplia distribución en la Argentina, si bien no se dispone de información sobre su prevalencia.

\section{Análisis de los costos}

Estimamos el total de casos de diarrea asociados con rotavirus cada año en la Argentina y también el número de pacientes ambulatorios y hospitalizados a fin de determinar los costos aproximados de la diarrea por rotavirus a nivel nacional (cuadro 3 y figura 4). El tamaño de la cohorte de los niños en riesgo se calculó sobre la base del Censo Nacional de Población y Vivienda de 1991 (41). El porcentaje de hospitalización en casos de diarreas asociadas con rotavirus fue calculado según los datos obtenidos en el estudio realizado en el Hospital de Niños de Buenos Aires (25). Los datos epidemiológicos sobre la incidencia de diarreas en general, de diarreas por rotavirus y de diarreas que requieren hospitalización o atención médica se obtuvieron en el estudio prospectivo en Avellaneda (21, 22, 24).

Partiendo de que la incidencia de diarrea es de 2,1 casos por año-niño en niños argentinos de 0 a 23 meses, que se hospitaliza a $3,9 \%(21,22,24)$ de estos casos, y que la tasa de detección de rotavirus es de $19 \%$ en niños hospitalizados por diarrea (25), calculamos que 21000 de los 650000 niños argentinos que nacieron en 1991 terminarían siendo hospitalizados con diarreas por rotavirus en sus primeros 2 años de vida.

Enfocando la situación con una perspectiva ligeramente distinta, estimamos que la incidencia de diarreas asociadas con rotavirus era de 0,13 casos por año-niño en niños argentinos de 0 a 23 meses de edad $(21,22,24)$, que la proporción de los que requerían atención médica era de $50 \%$ y que $12,5 \%$ de las diarreas por rotavirus requerían hospitalización $(21,22,24)$. Sobre esta base, estimamos que el número anual de diarreas por rotavirus ascendía a 169000 casos, de los cuales

CUADRO 2. Serotipos de cepas de rotavirus en la Argentina

\begin{tabular}{|c|c|c|c|c|c|c|c|c|}
\hline \multirow[b]{2}{*}{ Referencia } & \multirow[b]{2}{*}{$\begin{array}{l}\text { Ciudad del } \\
\text { estudio }\end{array}$} & \multirow[b]{2}{*}{$\begin{array}{l}\text { Período } \\
\text { (años) }\end{array}$} & \multirow[b]{2}{*}{$\begin{array}{l}\text { Muestras estudiadas/ } \\
\text { serotipos identificados }\end{array}$} & \multicolumn{5}{|c|}{ Serotipos identificados } \\
\hline & & & & G1 & G2 & G3 & G4 & $\begin{array}{c}\text { Distinto de } \\
\text { G1-G4 }\end{array}$ \\
\hline Gómez (23) & Buenos Aires & 1983-1985 & $123 / 73$ & 28 & 13 & 10 & 18 & 4 \\
\hline Espul (29) & Mendoza & 1991-1992 & $45 / 43$ & 42 & - & 1 & - & - \\
\hline Castagnaro (30) & Tucumán & 1986-1991 & $126 / 114$ & 67 & 33 & - & 14 & - \\
\hline Total & & 1983-1992 & $294 / 230$ & 137 & 46 & 11 & 32 & 4 \\
\hline
\end{tabular}


CUADRO 3. Costo estimado y carga anual de morbilidad por diarrea rotavírica en la Argentina

\begin{tabular}{|c|c|c|}
\hline & & Argentina \\
\hline & No. & $\begin{array}{l}\text { Riesgo de un niño de } \\
0-23 \text { meses de edad }\end{array}$ \\
\hline Nacimientos & 650000 & \\
\hline Episodios de gastroenteritis por rotavirus & 169000 & $1 / 4$ \\
\hline Pacientes ambulatorios examinados & 84500 & $1 / 8$ \\
\hline Hospitalizaciones & 21000 & $1 / 31$ \\
\hline \multicolumn{3}{|l|}{ Costo } \\
\hline Hospitalizacióna & US\$ 25,2 millones & \\
\hline Atención ambulatoriab & US\$ 2,5 millones & \\
\hline Atención médica directa en total & US\$27,7 millones & \\
\hline
\end{tabular}

a Se estimó un promedio de 4 días de hospitalización a un costo diario de US\$300.

b Se estimó el costo del tratamiento en un promedio de US\$ 30 por caso.

FIGURA 4. Número estimado de episodios diarreicos, episodios de diarrea por rotavirus, y hospitalizaciones por rotavirus entre niños argentinos menores de 2 años de edad

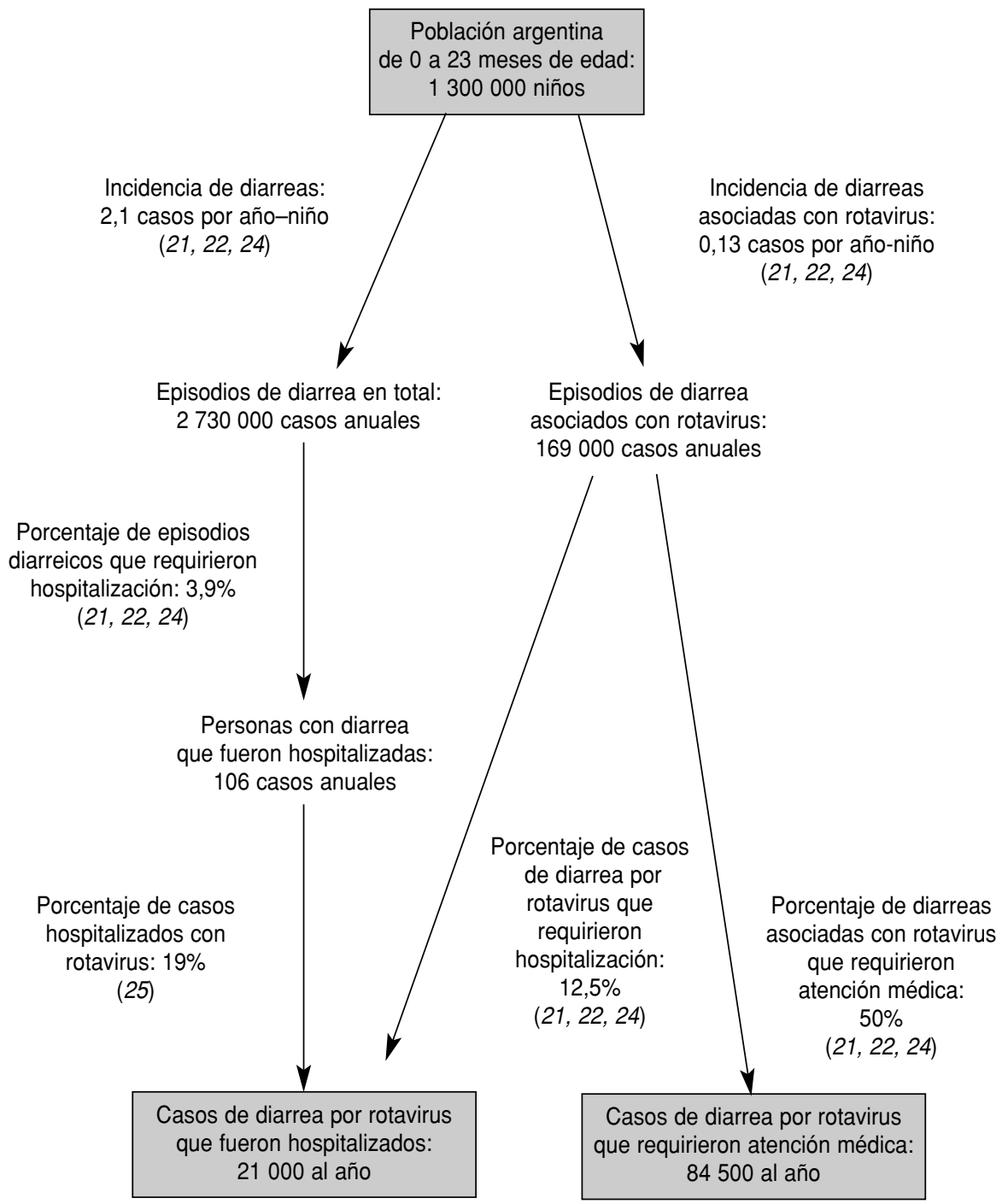

84500 requerían atención médica. En otras palabras, según estos cálculos, uno de cada cuatro niños argentinos sufre diarreas por rotavirus, uno de cada ocho busca atención médica para el tratamiento de esta y uno de 31 será hospitalizado por ese motivo durante sus primeros 2 años de vida.

El costo promedio de la internación se estimó en US\$300 al día, cifra basada en lo que cobran dos hospitales (el Hospital de Niños de Córdoba y el Hospital de Niños "Ricardo Gutiérrez" de Buenos Aires) al Servicio Nacional de Seguros de Salud de la Argentina. El costo promedio de atender de forma ambulatoria a un niño con diarrea por rotavirus se calculó en US\$30 por caso, estimación que se basó en el costo promedio de una consulta al médico o a una clínica, más transporte y tiempo que el padre/ tutor o encargado se ausentó de su trabajo. Al aplicarse nuestro cálculo del gasto diario en hospitalización, estimado en US\$300 al día, y un cálculo de un promedio de 4 días de internación más un costo en atención ambulatoria de US\$ 30 por caso, el costo de las diarreas asociadas por rotavirus en la Argentina se calculó en algo más de US\$ 27 millones anuales.

\section{DISCUSIÓN}

La perspectiva de tener disponible una vacuna contra rotavirus en un futuro próximo nos alentó a revisar los estudios epidemiológicos de las diarreas por rotavirus en la Argentina a fin de ayudar a definir la carga representada por la enfermedad y las necesidades de investigación, con miras a evaluar la posible utilidad de una vacuna en nuestro país. Los estudios examinados indicaron que en la Argentina los rotavirus son la causa más frecuente de diarrea grave en los niños, puesto que se identifican en una proporción mediana de $20 \%$ de los niños hospitalizados por diarrea. En general, los rotavirus tendieron a asociarse con enfermedades diarreicas relativamente graves y se hallaron más a menudo en niños hospitalizados con diarrea que en niños atendidos por la 
vía ambulatoria o incluidos en estudios de tipo comunitario. Sobre la base de los escasos estudios disponibles, calculamos que de los 650000 niños que nacieron en la Argentina en 1991, uno de cada ocho (84500) requirió atención médica y uno de cada 31 (21 000) fue hospitalizado por diarrea de origen rotavírico. A falta de un estudio concreto sobre el costo de la diarrea por rotavirus, hicimos un cálculo preliminar, según el cual los costos médicos de la enfermedad superaban los US\$ 27 millones anuales. En general, la introducción de una buena vacuna antirrotavírica en el programa nacional de inmunización para la infancia podría disminuir notablemente la carga de la enfermedad y ser de gran valor si la vacuna no fuera excesivamente cara. Obviamente, un estudio sobre su efectividad en función de los costos ayudaría a los planificadores a tomar una decisión.

Identificamos varias características de las diarreas por rotavirus en la Argentina, asociados con su frecuencia según la estación y su distribución por edades, que resultaron distintas de las que cabía esperar en un país desarrollado con un clima templado. Aunque se detectó el virus durante todo el año, los mayores niveles de detección se alcanzaron en invierno (de mayo a junio), cuando los virus se encontraron en cerca de $50 \%$ de los niños hospitalizados por diarrea que formaron parte de este estudio. Esta situación difiere mucho de la observada en Estados Unidos, donde la mayor detección de rotavirus coincide con el período del verano en que se produce el mayor número de hospitalizaciones por diarrea. Sin embargo, una investigación reciente en Estados Unidos de muchas infecciones aparentemente atribuibles a rotavirus durante el verano reveló que muchos de los casos diagnosticados se debían a resultados de laboratorio positivos falsos (42). Útil sería confirmar si fueron muchos los casos veraniegos que se debieron a rotavirus, a fin de aclarar si la diferencia observada entre los patrones estadounidense y argentino se puede atribuir a una transmisión más uniforme du- rante todo el año en los Estados Unidos o a errores de laboratorio.

En México, la transición del verano al invierno del pico máximo de las defunciones por diarreas infantiles se ha asociado con las importantes campañas nacionales cuya finalidad es combatir el cólera y otras diarreas bacterianas por medio de la cloración de las fuentes de agua potable y otras medidas (43). Otros estudios tal vez puedan dilucidar si los picos estivales que muestra la incidencia de diarreas bacterianas difiere mucho entre poblaciones de nivel socioeconómico alto y bajo en un país que tiene, como la Argentina, poca mortalidad infantil.

En el presente estudio, los niños argentinos se mostraron infectados por rotavirus a una edad más temprana que los niños en otros países desarrollados. Según los datos disponibles, más de $80 \%$ de los casos se presentaron en el primer año de vida y 35\% antes de los 6 meses de edad. Esta distribución de edad de los pacientes, que es más joven que la observada en otros medios propios de países desarrollados y de clima templado, podría asociarse con la transmisión de la infección durante el año entero. A consecuencia de este factor de edad, podría ser necesario aplicar una vacuna contra rotavirus más temprano, quizá administrando una dosis en el período neonatal. Puede ser, además, que solo se necesite protección durante un año y que el impacto máximo del programa de control sea mensurable en el primer año de la intervención.

Los estudios que aquí se examinan tienen muchas limitaciones y sus observaciones deben ser confirmadas. La gran variabilidad de las tasas de detección registradas en las distintas encuestas hospitalarias podría deberse a varios factores, entre ellos las fluctuaciones estacionales, la distribución de edad de los sujetos estudiados, la gravedad de su enfermedad y la sensibilidad de los ensayos utilizados. La falta de criterios comunes para el tamizaje realizado en estos estudios impidió que se investigaran tendencias geográficas o temporales, que podrían ser de gran interés. Por último, cabe señalar que nuestros cálculos de la carga de morbilidad representan una "libre estimación" preliminar de la magnitud del problema. Estos cálculos se basan en un pequeño número de encuestas que no han abarcado todo el país y tendrán que mejorarse utilizando datos nacionales sobre el número de hospitalizaciones o defunciones por diarrea infantil que no estaban disponibles cuando se hizo este análisis. Estos datos de carácter nacional serían puntos de referencia importantes, como también otros datos nacionales similares obtenidos mediante estudios realizados en los Estados Unidos y el Reino Unido $(4,44)$.

Ha servido de base este estudio para que en seis ciudades argentinas se iniciara una actividad de vigilancia a escala nacional en hospitales centinela. Los centros participantes utilizan una definición común para los casos, reúnen datos epidemiológicos comparables y examinan las muestras fecales con un mismo ensayo, cuya calidad estará sujeta al control del Laboratorio Nacional de Normas. Los datos obtenidos serán esenciales para obtener mejores estimaciones de la efectividad en función de los costos de un programa de vacunación contra los rotavirus y para comprender mejor las características específicas de la epidemiología de los rotavirus en la Argentina. Los datos proporcionados por el programa también podrían ser de importancia crítica a la hora de decidir si introducir o no una vacuna antirrotavírica en los programas corrientes de inmunización infantil y ciertamente serán útiles para observar las repercusiones de dicha inmunización en caso de que se lleve a la práctica.

Agradecimiento. Los miembros del Grupo para el Estudio de las Diarreas Virales son Nelda R. De Castagnaro y José A. Komaid (Instituto de Microbiología, Facultad de Bioquímica, Química y Farmacia, Universidad Nacional de Tucumán), Carlos Espul y Héctor Cuello (Laboratorio de Virología, Hospital Central de Mendoza), Jorge A. Gómez y Karin Bok (Labo- 
ratorio de Gastroenteritis Virales, Departamento de Virología, Instituto Nacional de Enfermedades Infecciosas, Ministerio de Salud y Acción Social de la Nación), Miguel O. Giordano y Silvia Nates (Instituto de Virología
"Dr. J. M. Vanella", Facultad de Ciencias Médicas, Universidad Nacional de Córdoba), Ana Borsa (Laboratorio de Virología, Hospital de Niños de La Plata), Saúl Grinstein (Laboratorio de Virología, Hospital de Niños "Ricardo
Gutiérrez" de Buenos Aires) e Isabel Miceli (Dirección de Epidemiología, Ministerio de Salud y Acción Social de la Nación).

\section{REFERENCIAS}

1. World Bank. World Bank Development Report 1993: Investing in Health. En: New York: Oxford University Press; 1993. p. 329.

2. Bishop RF, Davidson GP, Holmes IH, Ruck BJ. Virus particles in epithelial cells of duodenal mucosa from children with viral gastroenteritis. Lancet 1973;1:1281-1283.

3. De Zoysa I, Feachem RV. Intervention for the control of diarrhoeal diseases among young children: rotavirus and cholera immunization. Bull World Health Organ 1985;63: 569-583.

4. Glass RI, Kilgore PE, Holman RC, Jin S, Smit JC, Woods PA, et al. The epidemiology of rotavirus diarrhea in the United States: surveillance and estimates of disease burden. $J$ Infect Dis 1996;174 (supl. 1):S5-11. (Presentado en el $5^{\circ}$ Taller sobre Vacunas Antirrotavíricas, Atlanta, Georgia, 16 a 17 de octubre de 1995).

5. Ward RL, Bernstein DI, por el US Rotavirus Vaccine Efficacy Group. Protection against rotavirus disease after natural rotavirus infection. J Infect Dis 1994;169:900-904.

6. Bernstein DI, Sander DS, Smith VE, Schiff, GM, Ward RL. Protection from rotavirus reinfection: 2 year prospective study. J Infect Dis 1991;164:277-283.

7. Velásquez FR, Matson DO, Calva JJ, Guerrero ML, Morrow AL, Carter-Campbell S, et al. Rotavirus infection in infants as protection against subsequent infections. New Engl J Med 1996;335:1022-1028.

8. Bishop RF, Barnes GL, Cipriani E, Lund JS. Clinical immunity after neonatal rotavirus infection: a prospective longitudinal study in young children. New Engl J Med 1983;168, 309:72-76.

9. Bhan MK, Lew JF, Sazawal S, Das BK, Gentsch JR, Glass RI. Protection conferred by neonatal rotavirus infection against subsequent diarrhea. J Infect Dis 1993;168:282-287.

10. Hardy D. Epidemiology of rotaviral infection in adults. Rev Infect Dis 1967;9:461-469.

11. Wyatt RG, James WD, Bohl EH, Theil KW, Saif LJ, Kalica AR, et al. Human rotavirus type 2: cultivation in vitro. Science 1980;207: 189-191.

12. Kapikian AZ, Vesikari T, Ruuska T, Madore HP, Christy C, Dolin R, et al. An update on the "Jennerian" and modified "Jennerian" approach to vaccination of infants and young children against rotavirus disease. Adv Exp Med Biol 1992;327:59-69.

13. Clark HF, Offit PA, Ellis RW, Eiden JJ, Krah D, Shaw AR, et al. The development of multivalent bovine rotavirus (strain WC3) reassor- tant vaccine for infants. I Infect Dis 1996;174 (suppl. 1):S73-80.

14. Lombardi DG, Roseto AM, Stamboulian D, Barrera Oro JG. Virus of infantile gastroenteritis in Argentina. Lancet 1975;2:1311-1314.

15. Muchinik FR, Grinstein S. Rotavirus infection in children hospitalized for diarrhoea in Argentina. Ann Trop Paediatr 1980,1;167-173.

16. Muchinik FR, Grinstein S. Rotavirus in Buenos Aires, Argentina. Intervirology 1980; 13;253-256.

17. Plaza A, Grinstein S, Muchinik G, Valvaro M, Gómez JA. Estudio clínico y epidemiológico de la diarrea por rotavirus en la infancia. Arch Argent Pediatr 1982;80:289-308.

18. Komaid JA, Caillou MSL, Suárez AM, Zamora AM, Castagnaro NR. Detección de rotavirus no-A en Tucumán. Rev Argent Microbiol 1991; 23:172-174.

19. Sorrentino A, Schodeller EA, Bellinzoni R, Muchinik G, La Torre JL. Detection of an atypical rotavirus associated with diarrhoea in Chaco, Argentina. Trans $R$ Soc Trop Med Hyg 1986;80:120-122.

20. Komaid JA, Caillou SL, Suárez AM, Castagnaro N. Electroserotipos del ARN de rotavirus durante cuatro años de estudio de gastroenteritis en Tucumán. Rev Argent Microbiol 1990;22:123-129.

21. Grinstein S, Gómez JA, Bercovich JA, Biscotti EL. Epidemiology of rotavirus infection and gastroenteritis in prospectively monitored Argentine families with young children. Am J Epidemiol 1989;130:300-308.

22. Gómez J, Bercovich A, Alvarez A, Garrido D, Grinstein S. Seroepidemiology of human rotaviruses in a community of the Avellaneda district, Province of Buenos Aires. Rev Argent Microbiol 1990;22:182-191.

23. Gómez J, Estes MK, Matson DO, Bellinzoni R, Álvarez A, Grinstein S. Serotyping of human rotavirus in Argentina by ELISA with monoclonal antibodies. Arch Virol 1990;112:249-259.

24. Gómez JA, Bercovich J, Biscotti E, Siniawski S, Sternberg L, Montesi A, et al. Diarrea por rotavirus: estudio prospectivo de 49 familias del partido de Avellaneda, provincia de Buenos Aires. Arch Argent Pediatr 1967;85: 139-149.

25. Gómez JA, Biscotti EL, Grinstein S. Epidemiology of human rotaviruses in Argentina as determined by RNA genome electrophoresis. Intervirology 1986;26:174-180.

26. Gómez JA, Bercovich JA, Grinstein S. Comparison of enzyme immunoassay, counterim- munoelectrophoresis, and polyacrylamide gel electrophoresis for the diagnosis of rotaviruses. Rev Argent Microbiol 1985;17:111-114.

27. Giordano MO, Depetris AR, Nates SV. Estudio retrospectivo de la circulación de electroserotipos de rotavirus humano en la ciudad de Córdoba, Argentina, 1979-1989. Rev Argent Microbiol 1995;27:1-10.

28. Giordano MO, Basnec SN, Nates SV, Bennun F, Depetris AR. Rapid techniques for diagnostic and epidemiologic studies of rotavirus infection. J Virol Methods 1991;35:59-63.

29. Espul C, Cuello H, Navarta LM, Mamani N, O'Ryan M. Characterization of antigenic types of circulating rotaviruses in Mendoza, Argentina, based on typing of the external VP7 capsid protein. Acta Gastroenterol Latinoam 1993;23:211-216.

30. Castagnaro NCR, Komaid JA, Caillou MSL, Zamora AM, Naval MSM, Suárez AM. Variación temporal de electroserotipos y serotipos de rotavirus humanos en el noroeste argentino. Acta Bioquim Clin Latinoam 1994; 28:385-391.

31. Castagnaro NR, Caillou SL, Komaid JA, Díaz $\mathrm{N}$, Suárez AM, Zamora AM. Infección rotaviral detectada en la sala de hidratación del Hospital del Niño Jesús. Cienc Med 1991;6: 27-33.

32. Castagnaro NR, Komaid JA, Suárez AM, López de Caillou S, Raya JM. Detección de rotavirus (RV) en casos de gastroenteritis infantil (GEI) en Tucumán: comparación de las técnicas de enzimoinmunoensayo (ELISA) y electroforesis del RNA vírico (PAGE). Rev Fac Med Tucumán 1987;19:3-5.

33. Caillou SL, Komaid JA, Suárez A, Castagnaro $\mathrm{AE}$, Zamora A, Castagnaro NR. Búsqueda de rotavirus en neonatos. Cienc Med 1993;8: 319-325.

34. Basnec SN, Giordano MO, Bennun FR, Nates SV, Bergara M, Depetris AR. Detection of two atypical rotavirus in the province of Misiones, Argentina. Acta Virol 1991;35:408-412.

35. Aramayo I, Galanternik L, Botto L, Eldid ME, Gómez JA, Grinstein S, et al. Agentes patógenos entéricos aislados en niños con diarrea aguda asistidos en el Hospital de Niños "Ricardo Gutiérrez". Arch Argent Pediatr 1987;85:181-189.

36. Caillou MSL, Komaid JA, Zamora AM, Figueroa T, Castagnaro NCR. Comparación de los patrones electroforéticos de rotavirus detectados en Tucumán y Jujuy. Universidad de Tucumán; 1991. (Informe inédito). 
37. Binstein N. Instituto Nacional de Enfermedades Infecciosas; 1995. (Datos inéditos).

38. Borsa A. Laboratorio de Virología, Hospital de Ninos de La Plata; 1995. (Datos inéditos).

39. Gómez JA; 1991. (Informe inédito).

40. Taniguchi K, Urasawa T, Morita Y, Greenberg HB, Urasawa S. Direct serotyping of human rotavirus in stools using serotype 1-, 2-, 3-, and 4-specific monoclonal antibodies to VP7. J Infect Dis 1987;155:1159-1166.

41. Instituto Nacional de Estadística y Censos. Censo Nacional de Población y Vivienda. Buenos Aires: INDEC;1991. p. 101.
42. LeBaron CW, Allen JR, Hebert M, Woods PA, Lew JF, Glass RI y laboratorios que participan en el Sistema Nacional para la Vigilancia de Rotavirus. Outbreaks of summer rotavirus linked to laboratory practices. Pediatr Infect Dis I, 1992;11:860-865.

43. Gutiérrez G, Tapia-Conyer R, Guiscafré $\mathrm{H}$, Reyes H, Martínez H, Kumate J. Impact of oral rehydration and selected public health interventions on reduction of mortality from childhood diarrheal diseases in Mexico. Bull World Health Organ 1996;74: 189-197.
44. Ryan MJ, Ramsay M, Brown D, Gay NU, Farrington $\mathrm{CP}$, Wall PG. Hospital admissions attributable to rotavirus infection in England and Wales. I Infect Dis 1996;174 (suppl. 1): S12-18.

Manuscrito recibido el 4 de noviembre de 1996 y aceptado para publicación en versión revisada el 20 de agosto de 1997.

ABSTRACT Rotavirus is the most common cause of severe diarrhea in children worldwide, and vaccines currently being field-tested could be available for childhood immunization in several years. To assess the rotavirus disease burden in Argentina and the value of

Anticipating rotavirus vaccines: review of epidemiologic studies of rotavirus diarrhea in Argentina future national surveillance for the disease, we reviewed available data on rotavirus detections reported by published and unpublished studies conducted in nine Argentine cities and by a multicenter study. Data from these studies indicated that rotavirus was detected in $20 \%$ of 5226 specimens (within a range of $6 \%$ to $54 \%$ for different studies) from children hospitalized for diarrhea and in $9 \%$ of 6587 specimens (within a range of $5 \%$ to $22 \%$ for different studies) from children who were outpatients, members of mixed populations (hospitalized patients and outpatients), or survey subjects in community-based studies. The hospital data showed that while rotavirus was detected throughout the year, a peak occurred during the winter months (May-July), when up to half of the children with diarrhea were found positive for rotavirus. Attempted serotyping of 294 rotavirus-positive specimens for G-protein by three laboratories was successful in 230 cases $(78 \%)$; the resulting data indicated that serotype G1 was the most common (being present in $60 \%$ of the successfully serotyped specimens), followed by G2 (in 20\%), G4 (in 14\%), and G3 (in 5\%). Based on national data for Argentina, we estimate that in 1991 there were roughly 84500 rotavirus-associated outpatient visits ( 1 for every 8 births) and 21000 hospitalizations averaging 4 days in length ( 1 for every 31 births), all of which entailed direct medical costs estimated at US\$ 27.7 million. These preliminary data show that the rotavirus disease burden in Argentine children is extensive and could be decreased by a safe and effective vaccine. Further surveillance is needed to improve our understanding of the epidemiology and distribution of rotavirus strains in Argentina, to more accurately assess the cost-effectiveness of a rotavirus vaccine program, and to indicate what methods might best be used to monitor such a program's impact. 